\title{
Supplementary/Supporting Information
}

\section{A Sectioning and Database Enrichment Approach for Improved Peptide Spectrum Matching in Large, Genome-Guided Protein Sequence Databases}

Praveen Kumar, ${ }^{* 1+, 2 \ddagger}$ James E. Johnson, ${ }^{3 \S}$ Caleb Easterly, ${ }^{2 \ddagger}$ Subina Mehta, ${ }^{2 \ddagger}$ Ray Sajulga, ${ }^{2 \ddagger}$ Brook Nunn, ${ }^{4 l}$ Pratik D. Jagtap, ${ }^{2 \ddagger}$ Timothy J. Griffin ${ }^{\star 2 \ddagger}$

† Bioinformatics and Computational Biology, University of Minnesota-Rochester, Rochester, Minnesota 55904, United States

$\ddagger$ Biochemistry Molecular Biology and Biophysics, University of Minnesota-Twin Cities, Minneapolis, Minnesota 55455, United States

$\S$ Minnesota Supercomputing Institute, University of Minnesota-Twin Cities, Minneapolis, Minnesota 55455, United States

I| Department of Genome Sciences, University of Washington, Seattle, Washington 98195, United States

*Email: tgriffin@umn.edu. Phone: +1 (612) 624-5249

*Email: kumar207@umn.edu. Phone:+1 (612) 624-0381

$\underline{\text { Table of Content: }}$

Supplementary Section 1

Figure S1: Results using randomized sections on the SIHUMI data: Coefficient of Variation

Figure S2: Comparison of false PSMs and True PSMs

Page S-1

Figure S3: Meltwater: Increase in microbial PSMs

Page S-2

Figure S4: Meltwater: Unipept output comparison

Page S-3

Page S-4

Supplementary Section 2

Table S1: Decrease of True and False PSM identifications at a given target-decoy global FDR for the SIHUMI data set

Table S2: Pfu: PSMs identified

Page S-6

Supplementary Section 3: Instructions on accessing the database sectioning method Galaxy workflow on publicly available resources 


\section{Supplementary Section 1: Supplementary Figure}

Figure S1
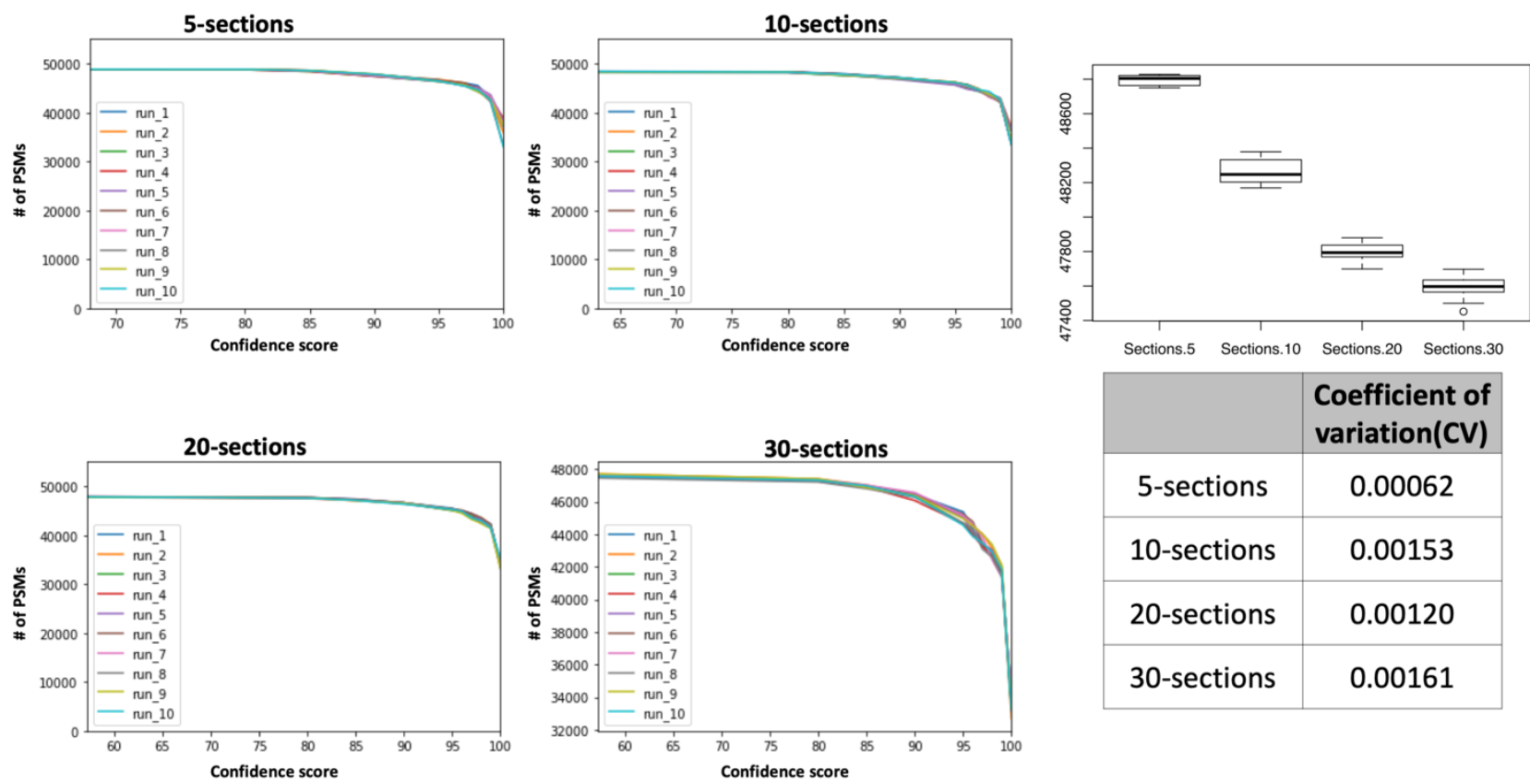

\begin{tabular}{|c|c|}
\hline & $\begin{array}{c}\text { Coefficient of } \\
\text { variation(CV) }\end{array}$ \\
\hline 5-sections & 0.00062 \\
\hline 10-sections & 0.00153 \\
\hline 20-sections & 0.00120 \\
\hline 30-sections & 0.00161 \\
\hline
\end{tabular}

Figure S1: SIHUMI PSMs: Sectioning involves randomly assigning protein sequences in each sectioned database bin. Shown here are results from running $10 \mathrm{such}$ randomizations for each 5, 10, 20, and 30 sections. Figures indicate that each randomization yielded very similar results, indicated by a low coefficient of variation shown in the table. 
Figure S2

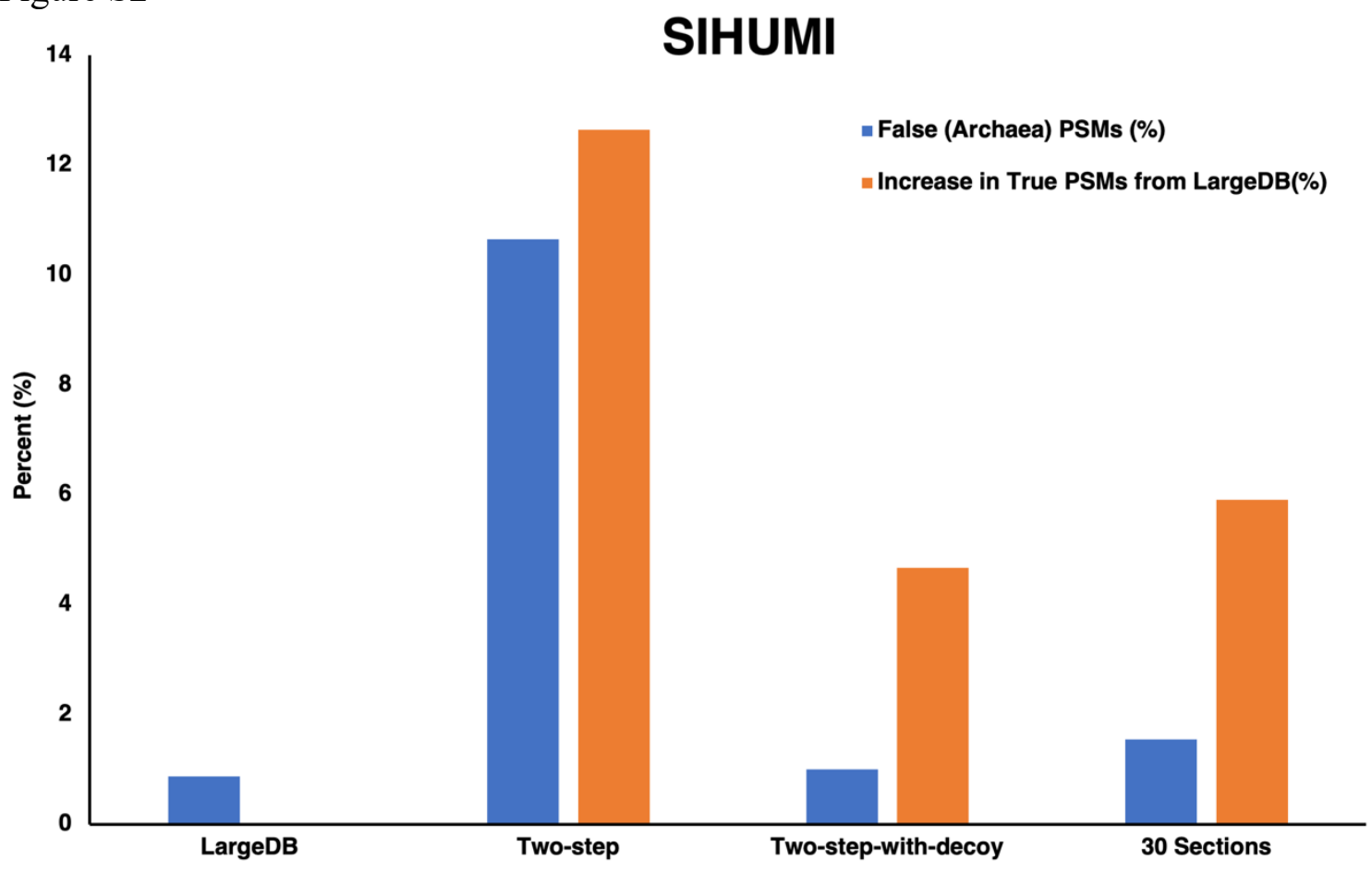

Figure S2: Comparison of Traditional, two-step, two-step-with-decoy, and sectioning methods based on percent false PSM identifications and increase in true PSMs in SIHUMI dataset. 
Figure S3

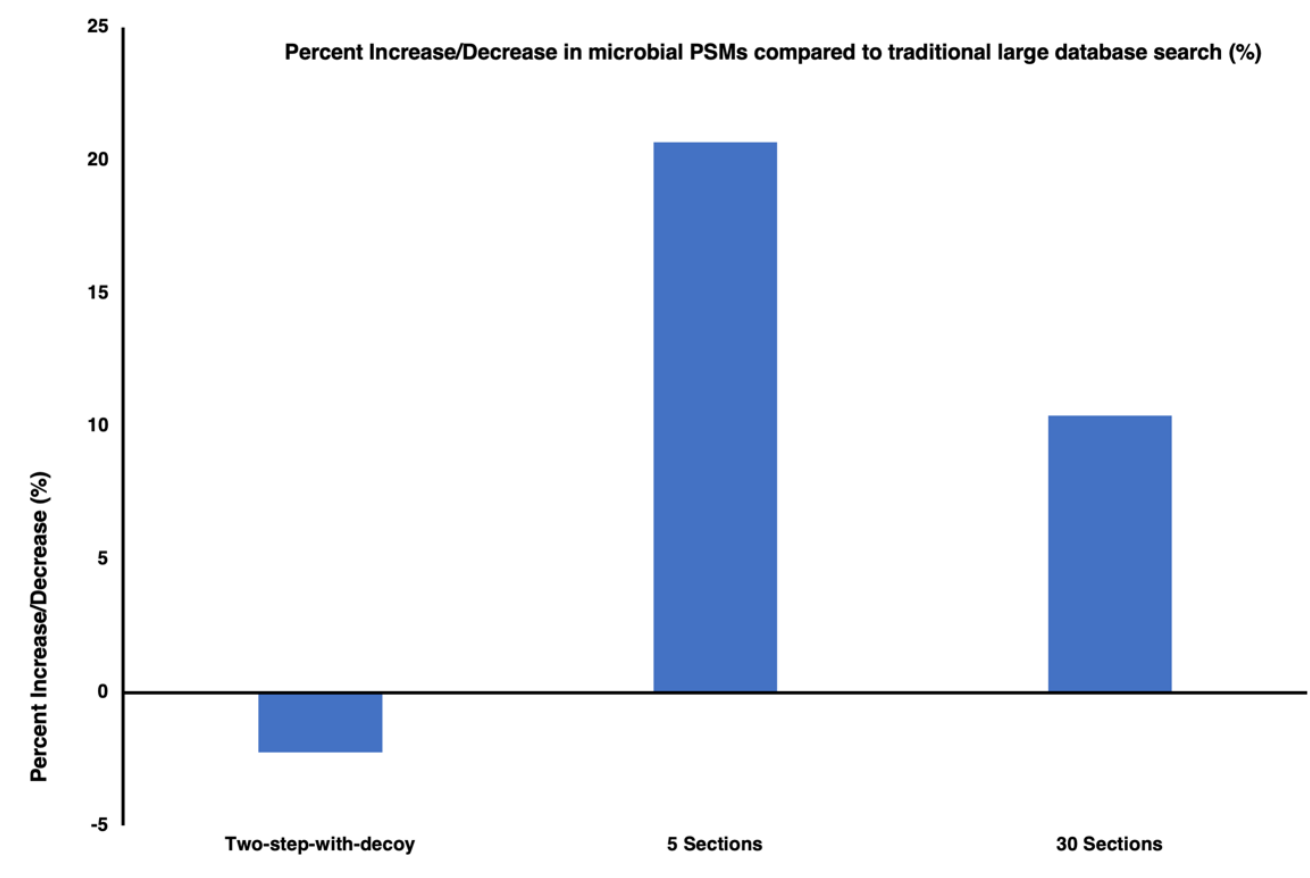

Figure S3: Percentage increase in the identification of microbial PSMs in meltwater datasets. Two-step-with-decoy method does not show any improvement whereas sectioning methods allow identification of more microbial PSMs compare to the traditional method. 
Figure S4

a'Protein EC

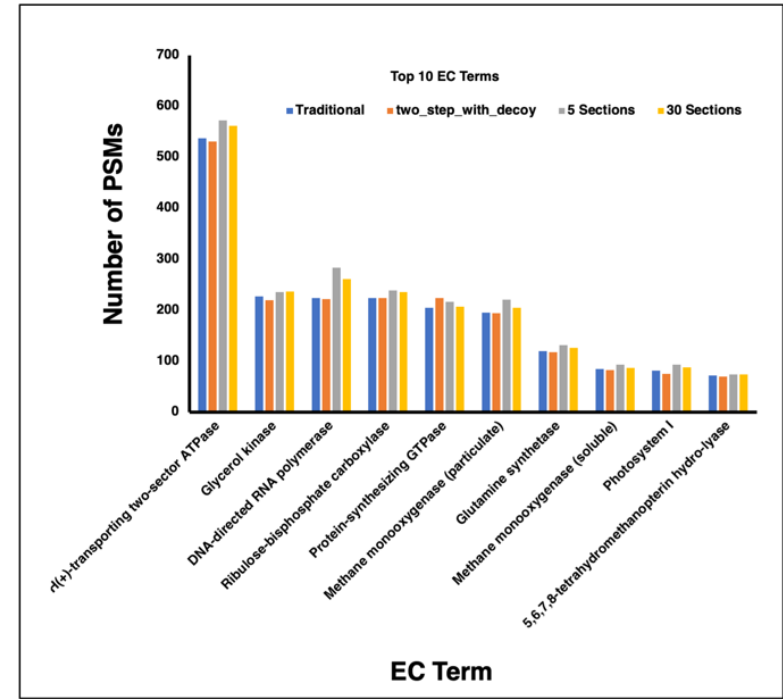

c

Biological Processes

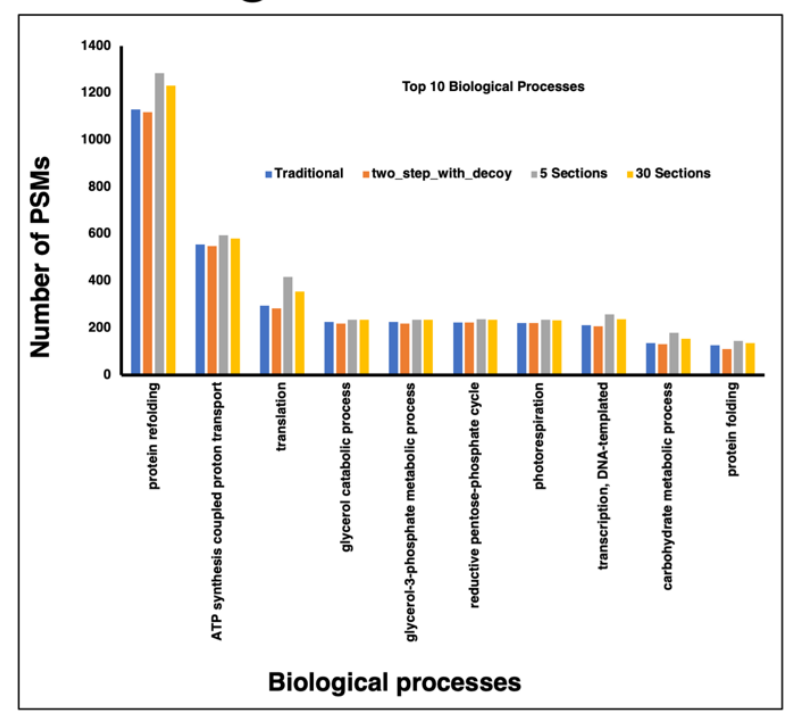

b

Taxonomy

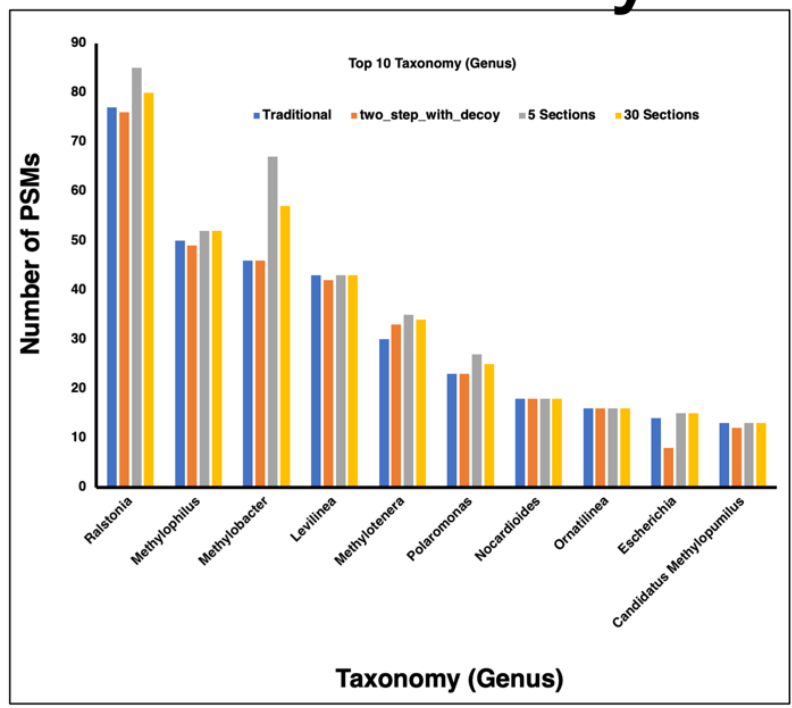

d

Molecular Functions

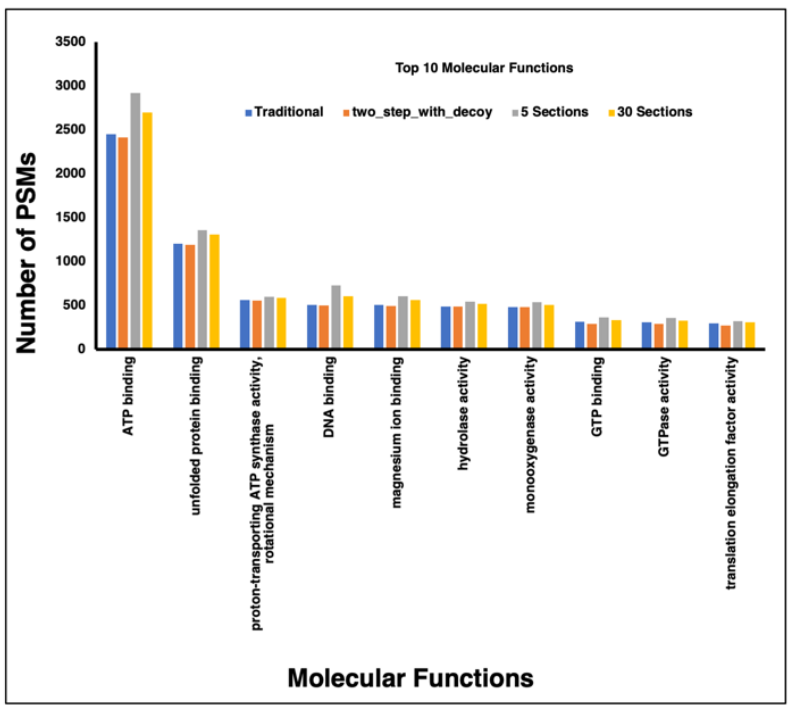

Figure S4: Meltwater dataset: Comparison of Unipept outputs from traditional database searching method, sectioning method (5 and 30 sections), and two-step-with-decoy method. In all cases, (a) Protein EC, (b) Taxonomy, (c) Biological Processes, and (d) Molecular Functions, the assignments from the Unipept analysis shows improved PSM assignments from the sectioning method. Two-step-with-decoy method performs similar to the traditional method providing no improvement in the PSM assignments. 
Supplementary Section 2: Supplementary Table

Table S1

\begin{tabular}{|c|c|c|c|c|c|}
\hline $\begin{array}{c}\text { Global } \\
\text { FDR } \\
\text { cutoff }\end{array}$ & $\begin{array}{c}\text { False } \\
\text { PSMs }\end{array}$ & $\begin{array}{c}\text { True } \\
\text { PSMs }\end{array}$ & $\begin{array}{c}\text { False positive } \\
\text { PSM (\%) }\end{array}$ & $\begin{array}{c}\text { (\%) Decrease in } \\
\text { False PSMs, } \\
\text { relative to the 1\% } \\
\text { global FDR level }\end{array}$ & $\begin{array}{c}\text { (\%) Decrease in } \\
\text { True PSMs, } \\
\text { relative to the 1\% } \\
\text { global FDR level }\end{array}$ \\
\hline $\mathbf{1}$ & 747 & 47581 & 1.55 & NA & NA \\
\hline $\mathbf{0 . 8}$ & 600 & 47245 & 1.25 & 19.68 & 0.71 \\
\hline $\mathbf{0 . 7 5}$ & 561 & 47176 & 1.18 & 24.90 & 0.85 \\
\hline $\mathbf{0 . 7}$ & 524 & 47065 & 1.10 & 29.85 & 1.08 \\
\hline $\mathbf{0 . 6 5}$ & 499 & 46943 & 1.05 & 33.20 & 1.34 \\
\hline $\mathbf{0 . 6}$ & 463 & 46771 & 0.98 & 38.02 & 1.70 \\
\hline
\end{tabular}

Table S1: Decrease of True and False PSM identifications at a given target-decoy global FDR for the SIHUMI dataset (30-sections). 
Table S2

\begin{tabular}{|c|c|c|c|c|c|c|}
\hline \multicolumn{7}{|c|}{ True PSMs } \\
\hline & $\begin{array}{c}2 \\
\text { Sections }\end{array}$ & $\begin{array}{c}5 \\
\text { Sections }\end{array}$ & $\begin{array}{c}10 \\
\text { Sections }\end{array}$ & $\begin{array}{l}\text { Traditional } \\
\text { (Large DB) }\end{array}$ & $\begin{array}{c}\text { Traditional 2- } \\
\text { Step }\end{array}$ & $\begin{array}{c}\text { Pfu } \\
\text { (Standard } \\
\text { search) }\end{array}$ \\
\hline instance 1 & 10598 & 10580 & 10635 & \multirow{10}{*}{10268} & \multirow{10}{*}{10621} & \multirow{10}{*}{10770} \\
\hline instance 2 & 10660 & 10593 & 10635 & & & \\
\hline instance 3 & 10592 & 10594 & 10618 & & & \\
\hline instance 4 & 10639 & 10611 & 10653 & & & \\
\hline instance 5 & 10624 & 10631 & 10601 & & & \\
\hline instance 6 & 10613 & 10616 & 10496 & & & \\
\hline instance 7 & 10653 & 10635 & 10604 & & & \\
\hline instance 8 & 10586 & 10629 & 10636 & & & \\
\hline instance 9 & 10632 & 10501 & 10635 & & & \\
\hline instance 10 & 10608 & 10627 & 10621 & & & \\
\hline \multicolumn{7}{|c|}{ False (Human) PSMs } \\
\hline & $\begin{array}{c}2 \\
\text { Section }\end{array}$ & $\begin{array}{c}5 \\
\text { Sections }\end{array}$ & $\begin{array}{c}10 \\
\text { Sections }\end{array}$ & $\begin{array}{l}\text { Traditional } \\
\text { (Large DB) }\end{array}$ & $\begin{array}{c}\text { Traditional 2- } \\
\text { Step }\end{array}$ & \\
\hline instance 1 & 273 & 186 & 108 & \multirow{10}{*}{102} & \multirow{10}{*}{360} & \\
\hline instance 2 & 191 & 154 & 156 & & & \\
\hline instance 3 & 234 & 171 & 116 & & & \\
\hline instance 4 & 157 & 149 & 116 & & & \\
\hline instance 5 & 233 & 116 & 157 & & & \\
\hline instance 6 & 249 & 169 & 99 & & & \\
\hline instance 7 & 193 & 142 & 144 & & & \\
\hline instance 8 & 300 & 170 & 126 & & & \\
\hline instance 9 & 213 & 150 & 150 & & & \\
\hline instance 10 & 217 & 126 & 152 & & & \\
\hline
\end{tabular}

Table S2: $P f u$ search results with true and false PSM identified by all the methods. 


\section{Supplementary Section 3: Accessing Sectioning Workflow}

The workflow can be accessed from the Galaxy server hosted publicly from Europe (https://z.umn.edu/sectioningWF_eu).

To save the workflow, click on the "+" sign found on the right top corner of the page.

The workflow requires two inputs - dataset collection of MGF files and protein sequences in FASTA format.

Core parameters and test dataset

1) Tool "Split file": set "Number of new files" to number of sections needed for analysis.

2) SearchGUI and PeptideShaker parameters should be checked and modified based on the dataset and its acquisition method. SearchGUI and PeptideShaker are used two times in the workflow. Modify parameters at both times as required.

3) Query Tabular (the one reading "Extended PSM Report" from PeptideShaker): The SQLite query can be modified depending on the optional score cutoff required.

"select distinct Proteins from PSM where Proteins not like '\%_REVERSED\%" and Confidence $>=0$ "

The "Confidence $>=0$ " is the most lenient and recommended cutoff. This would include Proteins identified from all PSMs. The Confidence value ranges from 0 to 100 and any value within this limit can be used. Though we recommend using lower value here.

4) A sample data is shared for testing the database sectioning method and can be accessed from a shared history on European Galaxy (https://usegalaxy.eu/u/galaxyp/h/sectioningsampleinputdata). 